Trinity University

Digital Commons @ Trinity

Psychology Faculty Research

Psychology Department

1979

\title{
Constructive Memory for Bizarre and Sensible Sentences
}

\author{
Paula T. Hertel \\ Trinity University, phertel@trinity.edu
}

H. C. Ellis

Follow this and additional works at: https://digitalcommons.trinity.edu/psych_faculty

Part of the Psychology Commons

Publication Details

Journal of Experimental Psychology: Human Learning and Memory

\section{Repository Citation}

Hertel, P.T., \& Ellis, H.C. (1979). Constructive memory for bizarre and sensible sentences. Journal of

Experimental Psychology: Human Learning and Memory, 5(4), 386-394. doi: 10.1037/0278-7393.5.4.386

This Article is brought to you for free and open access by the Psychology Department at Digital Commons @ Trinity. It has been accepted for inclusion in Psychology Faculty Research by an authorized administrator of Digital Commons@ Trinity. For more information, please contact jcostanz@trinity.edu. 


\title{
Constructive Memory for Bizarre and Sensible Sentences
}

\author{
Paula T. Hertel and Henry C. Ellis \\ University of New Mexico
}

\begin{abstract}
Sensible, interrelated sentences were presented with or without bizarre sentences that could be transformed to fit the context of the sensible sentences. Two experiments examined subjects' ability to recognize or recall both types of sentences, either immediately or after 2 weeks. Bizarre sentences were frequently recognized at immediate testing; they were generally unavailable at delayed recognition and were never recalled verbatim. In addition, results indicated that transformations of bizarre sentences were stored in memory but were not well incorporated within the structure for the sensible material. These findings are consistent with a constructive approach to memory. Finally, the results suggest that processing bizarre information can lead to more accurate recognition and recall of the sensible context.
\end{abstract}

The constructive approach to memory has emphasized that memory for an event can change qualitatively over the course of time. The approach has its roots in Bartlett's (1932) memory investigations in which subjects read folktales from unfamiliar cultures and reproduced them repeatedly over varying intervals of time (cf. Cofer, 1976). Bartlett drew two major conclusions concerning the processess affecting the observed memory changes: (a) Recall was reconstructed from schemata or representations of meaningful information that had been abstracted during the processing of the folktales and (b) subjects processed unusual portions of the folktales in an "effort after meaning," in an attempt to make the material more understandable and, therefore, easier to integrate into the overall meaning structure.

This research was supported by National Institute of Mental Health Grant $\mathrm{MH}-15142$ to the second author.

We wish to thank Elizabeth Loftus and Charles Cofer for their thoughtful comments on an earlier draft and John Schnizlein for helpful advice and the formulation of the conversion algorithm for $A^{\prime}$, based on Pollack and Norman (1964).

Requests for reprints should be sent to Henry C. Ellis, Department of Psychology, University of New Mexico, Albuquerque, New Mexico 87131.
Evidence for Bartlett's first conclusion, reconstruction from abstract representations, has been supplied by several closely related lines of research. The nature of what is remembered about meaningful material can be located on a continuum from verbatim to thematic information. For example, both recognition and recall errors in memory for sentence surface structure occur more frequently than meaning errors (Jarvella, 1971; Sachs, 1967). In addition, the meaning of individual sentences is forgotten more rapidly than paragraph gist or ideas expressed by more than one sentence (Bransford \& Franks, 1972; Dooling, Christiaansen, \& Keenan, Note 1). However, the tendency to rely on abstract information rather than verbatim information is partly determined by the type of material to be learned (Bransford \& Franks, 1972) and by task demands (Graesser \& Mandler, 1975).

Additional evidence that memory is reconstructed from holistic representations has emanated from experiments in inferential reasoning and context manipulations. The major thrust of these experiments is that extra experimental knowledge (Bransford, Barclay, \& Franks, 1972; Sulin \& Dooling, $1974)$ or related experimental materials (Bransford \& Johnson, 1972; Loftus, 1975 ; 
Loftus, Miller, \& Burns, 1978) contribute to changes in memorial performance.

The great majority of research in this area has emphasized the constructive processing of sensible material. In contrast, little support has been found for Bartlett's (1932) conclusion that bizarre information is omitted or transformed to make it more comprehensible. Although Bartlett did find that subjects frequently omitted portions that were difficult to understand, he did not report data concerning immediate memory for the bizarre portions. In addition, the use of a reproductive memory task may have biased subjects to write well-integrated, sensible stories, explaining or omitting unusual elements that may have been remembered. Finally, repeated attempts to recall involve the obvious problem of memory for the results of earlier efforts.

The following experiments were designed to test Bartlett's (1932) conclusions concerning memory for the bizarre. Bizarre sentences were constructed by violating rules of language usage, specifically by having inanimate objects perform actions of which they are incapable; an example is "The hot dogs prepared the woman." The bizarre sentences were presented along with sensible sentences that provided a context for possible transformations of the bizarre. For example, hearing that it was dinner time, the woman was dutiful, hot dogs were steaming, and children were hungry establishes a context for transforming the bizarre information into the idea that the woman prepared the hot dogs. A constructive approach to memory predicts that bizarre information should be, for the most part, forgotten at delayed testing due to the difficulty of constructing accurate semantic representations or to the difficulty of combining verbatim representations of the bizarre and the sensible material into a meaningful whole. In addition, strong evidence for integrated memory would be provided by the transformation of the bizarre elements into sensible information more consonant with the interrelated context. The latter possibility is the phenomenon referred to by Bartlett as effort after meaning. Finally, we expected to find that the sensible context was stored in an integrated fashion. In summary, these experiments focus on two principal issues: (a) the temporal course of memory for bizarre information and (b) the effect of the bizarre information on memory for the sensible context.

\section{Experiment 1}

The first experiment employed a modification of Bransford and Frank's (1972) paradigm for demonstrating integrated memory. This paradigm was chosen because it involves the presentation of single sentences. Presenting single sentences should decrease the bias against responding to strange elements, which may exist with prose material. In addition, the single sentences are solely recombinations of simple ideas contained in one complex idea. Bransford and Franks have shown that subjects construct the complex idea (which is never presented during acquisition) as the most efficient means of encoding the experimental material. Therefore, in this experiment, the sensible material, presented as a list of such sentences, should be stored in a unified fashion and should provide an appropriate context for transforming the bizzare.

The procedure consisted of an acquisition phase, during which subjects wrote each sentence after hearing it, and a recognition phase, during which subjects attempted to detect previously presented sentences out of a list of old sentences combined with several types of distractors, including transformations of the bizarre. The acquisition list either included the bizarre sentences (bizarre condition) or did not (control condition), and recognition testing occurred either immediately or after a 2 -wk. delay.

Recognition of bizarre sentences was expected to be excellent at immediate testing, demonstrating a lack of bias against processing the unusual. Second, given a high level of immediate recognition, bizarre information was expected to be unavailable at delayed testing. In addition, bizarre-acquisition subjects were predicted to be poorer than control-acquisition subjects at rejecting 
transformations of the bizarre, compared to nontransformational distractors.

\section{Method}

Subjects. Forty-eight volunteers from introductory psychology classes at the University of New Mexico served as subjects ( 12 in each condition) and received class credit. Groups of 4-8 subjects were randomly assigned to acquisition conditions, and subjects within groups were randomly assigned to test conditions.

Materials. Two idea sets, or complex propositions, were invented and are presented in Table 1. Each idea set may be broken into six simple ideas, and sentences may be constructed that contain from one to four of the simple ideas within each idea set.

For both acquisitions lists, 16 of these sentences were constructed so as not to include the main idea of the idea set ("The woman prepared hot dogs" and "The grandfather comforted the girl"). Constraints of equal frequency of idea set and sentence length (from one to four simple ideas) were observed. Each simple idea occurred an equal number of times and was just as likely to occur in a short sentence as in a long one. Examples of two-idea, sensible acquisition sentences are also presented in Table 1 . The two acquisition lists differed only with respect to the bizarre sentences in Table 1 . The bizarre list contained these sentences in Positions 5 and 12, whereas the control list contained two extra one-idea sentences in the corresponding positions. The remaining list positions were randomly determined, with the restriction that sentences from the same idea set did not occur consecutively. A total of 18 sentences occurred on each list.

The recognition list contained the two bizarre sentences, eight old sensible sentences, and eight sentences from each of four distractor types. In all cases, the eight sentences were comprised of two sentences within each sentence length (1-4), one of the two from each idea set (except for noncase distractors, in which case sentences contained ideas from both idea sets).

The two-idea sentences for each distractor type are presented in Table 1. New distractors were constructed just like the sensible-acquisition sentences; however, they were not presented until recognition testing. (Similarly constructed sentences were used by Bransford and Franks, 1972, to demonstrate loss of memory for exact wording.) Transformed sentences all included the main idea of the idea set, which was never presented during acquisition. Inference sentences all included an alternate way of stating the main idea; the verbs cooked and helped were consistently used in place of prepared and comforted. These sentences served as controls for the possibility. that transformed sentences might be responded to solely on the basis that they contain logical inferences. Finally, noncase sentences contained words or ideas from both idea sets and were included as controls for semantic accuracy.

Selection of the 42 sentences was randomized for each of four recognition list orders; idea sets alternated. The assignment of list order was counterbalanced within experimental conditions.

Procedure. Instructions and materials for the acquisition phase were presented on a cassette recorder. Subjects were told that they would hear a list of sentences and that their task was to

Table 1

Acquisition and Recognition Materials for Experiment 1

Idea set

Item At dinnertime the dutiful woman prepared steaming hot dogs for the hungry children.

The protective grandfather rushed outside to comfort the little girl who fell off her bike and was crying.

Acquisition sentences

Sensible At dinnertime the children were hungry. The little girl was crying.

Bizarre The hot dogs prepared the woman.

\section{Recognition distractors}

New The steaming hot dogs were for the children. The grandfather was protective and the girl fell off her bike.

Transformed At dinnertime the woman prepared hot dogs.

The grandfather comforted the girl who fell off her bike.

Inference The woman cooked hot dogs for the children The protective grandfather helped the girl. Noncase The dutiful woman rushed outside. The girl was little and the children were hungry.

Note. Examples of sensible acquisition sentences and distractors are all two ideas in length, although lengths of one to four were employed in the experiment. 
listen to each sentence, name the color of a card held by the experimenter, write the sentence, and fold the paper down to cover the sentence. Two practice trials were given. Sentence presentation rate was controlled by the experimenter to allow an interval long enough for all subjects to write the sentences; the interval varied from approximately 15 to $30 \mathrm{sec}$. Following the acquisition phase, half of the subjects in each group were randomly selected to return in 2 wk. and were not told what to expect on return.

The recognition phase was administered to groups of two to four subjects. Response sheets were numbered from 1 to 42 , with the words YES No next to each number. Subjects were instructed to judge whether each recognition sentence had been presented previously in the experiment by circling the appropriate word. The list was presented on tape, with a 5-sec pause between sentences.

\section{Results and Discussion}

Preliminary analyses found no reliable interactions with idea set; therefore, the data were collapsed across this variable. Table 2 presents the hit rates or false alarm rates for each type of recognition sentence. For analyses concerning memory for bizarre sentences, the hit rates for these sentences were compared to those for old sensible sentences. Second, to analyze detection differences among distractor types, false alarm rates and the hit rates for old sentences were converted into $A^{\prime}$ statistics, which are comparable to percentage correct in a two-alternative; forced-choice task (Green \& Swets, 1974, Appendix III, 3.3). Table 3 presents $A^{\prime}$ cell means for each distractor type. A value of .5 represents the inability to detect differences between the distractors and the old sensible sentences.
Memory for the bizarre. The relevant data in Table 2 are the proportion of yes responses to bizarre and old sentences that were made by subjects in the bizarre-immediate and bizarre-delay conditions. Clearly, the number of positive responses to old sensible sentences remained at a substantial level over the delay interval, whereas those to bizarre sentences decreased dramatically, $F(1,44)=23.70, M S_{\mathrm{e}}=4.57, p<.001$. Although fewer bizarre sentences were presented on the acquisition list, subjects recognized them as well as old sentences when they were tested immediately, $F(1,11)=$ $.90, M S_{\mathrm{e}}=9.24$.

Transformations of the bizarre. The means in Table 3 indicate that rejection of transformed sentences decreased over time for both acquisition conditions, $F(1,44)=$ 19.00, $M S_{\mathrm{e}}=.012, p<.001$. In addition, control conditions were reliably better than bizarre conditions in rejecting the transformed distractors, $F(1,44)=4.85, M S_{\mathrm{e}}=$ $.012, p<.033$. More importantly, at delayed testing the difference between rejection of transformed and inference distractors was greater for the bizarre condition than for the control condition, $F(1,44)=8.47, M S_{\mathrm{e}}$ $=.016, p<.006$. Therefore, at delayed testing, only the responses of subjects hearing bizarre input indicated a memory bias for sensible restatements of the bizarre (transformed distractors), which was greater than any tendency to respond to statements implied by the sensible input sentences (inference distractors).

Results that were somewhat unexpected

Table 2

Proportion of Yes Responses for Each Type of Recognition Sentence

\begin{tabular}{llllll}
\hline & & \multicolumn{4}{c}{ Condition } \\
\cline { 3 - 6 } Sentence type & $n$ & $\begin{array}{c}\text { Bizarre } \\
\text { immediate }\end{array}$ & $\begin{array}{c}\text { Bizarre } \\
\text { delay }\end{array}$ & $\begin{array}{c}\text { Control } \\
\text { immediate }\end{array}$ & $\begin{array}{c}\text { Control } \\
\text { delay }\end{array}$ \\
\hline Bizarre & 2 & .63 & .17 & .00 & .00 \\
Old & 8 & .73 & .80 & .74 & .85 \\
New & 8 & .57 & .77 & .72 & .84 \\
Transformed & 8 & .29 & .61 & .14 & .62 \\
Inference & 8 & .21 & .35 & .16 & .54 \\
Noncase & 8 & .00 & .11 & .01 & .16 \\
\hline
\end{tabular}

Note. $n=$ the number of each type on the recognition list. 
Table 3

Mean $A^{\prime}$ for Each Type of Distractor Sentence in Each Condition

\begin{tabular}{lccccc}
\hline & \multicolumn{5}{c}{ Condition } \\
\cline { 2 - 5 } & Distractor type & $\begin{array}{c}\text { Bizarre } \\
\text { immediate }\end{array}$ & $\begin{array}{c}\text { Bizarre } \\
\text { delay }\end{array}$ & $\begin{array}{c}\text { Control } \\
\text { immediate }\end{array}$ & $\begin{array}{c}\text { Control } \\
\text { delay }\end{array}$ \\
New & .64 & .52 & .53 & .50 \\
Transformed & .80 & .68 & .89 & .73 \\
Inference & .83 & .81 & .87 & .71 \\
Noncase & .93 & .91 & .93 & .91 \\
\hline
\end{tabular}

Note. $\mathrm{A}^{\prime}$ was computed from the hit rate for the eight old sentences, compared to the false-alarm rate for the eight sentences from each distractor type. A score of .5 represents the inability to differentiate between old sentences and distractors.

were the relatively low rejection levels for transformed and inference distractors by control-delay subjects. These results may indicate that both types of sentences were easily inferred from the acquisition material. In general, as memory for detail presumably declined over time, all subjects' responses increasingly reflected logical inferences. The inferences may have been stored at acquisition and easily rejected when testing was immediate, due to still excellent memory for detail. Or, the inferences may have occurred at delayed testing, due to the nonavailability of specific input information. However, it is clear that subjects in the bizarre-acquisition condition differentiated possible inferences based on their exposure to bizarre sentences. They retained some information as long as 2 wk following acquisition, which allowed them to respond positively to transformed sentences and to mostly reject bizarre sentences. This pattern of responding may indicate transformational encoding.

New and noncase sentences. All subjects were uniformly superior at rejecting noncase sentences, as in other experiments using this paradigm (Bransford \& Franks, 1972). Furthermore, Table 2 shows that for most conditions, the difference in proportion of yes responses between old and new sensible sentences was unreliable, replicating Bransford and Franks's findings, except for the bizarre-immediate condition. The difference in proportion of yes responses was reliably greater for this condition than for the control-immediate condition, $F(1,44)=4.36$,
$M S_{\mathrm{e}}=1.62, p<.041$. But since the $A^{\prime}$ data (Table 3) did not reflect a reliable difference between bizarre-immediate and control-immediate conditions in the rejection of new sentences, the interpretation is difficult. A plausible interpretation is that the occurrence of bizarre sentences inculcated a more conservative bias in responding to recognition sentences.

Additional support for an effect of bizarre information on context accuracy was provided by examining sentences written during the acquisition task for potential differences between acquisition conditions. Sentences copied following the occurrence of the first bizarre sentence (Position 5) were scored for the number of errors in surface structure that they contained (omissions or additions of simple ideas, phrase-order reversals, and other order confusions). Errors were twice as frequent in the control condition as in the bizarre condition (totals of 21 and 10 , respectively). A chi-square analysis was performed by categorizing the performance of each subject according to acquisition condition and the presence or absence of surface-structure error $\left(x^{2}\right.$ corrected for continuity $=5.34, p<.025)$. In both conditions, very few errors occurred prior to the first bizarre sentence, and bizarre sentences were copied accurately.

\section{Experiment 2}

The results from the first experiment demonstrated that omissions and transformations of bizarre information were characteristic of recognition performance. However, bi- 
zarre-delay subjects could often reject transformed sentences; for example, there was a substantial difference between the delayed rejection of these sentences and new sentences. Furthermore, control-delay subjects tended to falsely recognize both types of inferences. These results indicated that providing recognition distractors may have constrained memory performance. Therefore, a cued-recall task was employed in Experiment 2 to allow for the expression of unique transformations and to compare levels of inference production across conditions.

\section{Method}

Subjects. Thirty-one students participated in the experiment. The acquisition materials were presented to groups of 7-9 subjects, and the size of the test groups ranged from 3 to 6 . Groups were randomly assigned to acquisition conditions, and subjects within groups were randomly assigned to testing conditions.

Materials. The acquisition lists were identical to those in Experiment 1. All response sheets for cued recall followed the format of three key words from one idea set typed across the top of the page, with nine numbered spaces below. For the idea set concerning the woman, cuing words were woman, hot dogs, and children, and for the second set, they were grandfather, girl, and bike.

Procedure. The acquisition phase and retention intervals duplicated Experiment 1. The testing phase began with the distribution of response sheets, and the following instructions were presented on tape :

Your task is to recall the nine sentences you have heard in this experiment which contained one or more of the words at the top of the page, and to write these sentences in the spaces provided. Each sentence you write must contain at least one of the words you see, and you must write all nine. If you have great difficulty remembering a sentence, write a close approximation to what it might have been.

Instructions mentioned approximations so that subjects tested at delay would consider the task more reasonable. After the $10 \mathrm{~min}$. allowed for first recall, the second response sheet was distributed, and the instructions were repeated. Order of ideaset recall was counterbalanced within each testing group.

\section{Results and Discussion}

Scoring. Protocol sentences were examined for possible classification into one of four categories. Sentences were judged as belonging to the idea-set category if they contained simple ideas or combinations of simple ideas within the idea set and did not include elaborations; word substitutions were allowed as long as they preserved the meaning of the ideas. The inference category was employed for sentences stating a relationship between the subject of the first clause and the subject of the second clause of each full idea set, such that the subject of the first clause acted on the subject of the second clause (for example, "The woman made hot dogs for the hungry children."). Other intrusions were allowed, and they occurred infrequently. The elaboration category was reserved for all sentences containing information that could not be directly inferred from either idea set, such as the notions that the children were playing, the grandfather was sitting on the porch, or the hot dogs were in the oven. This category was not used for sentences containing the inferences discussed previously. Finally, the bizarre category was intended for verbatim reproductions of the bizarre sentences, plus any sentences judged to be transformations of bizarre sentences.

Other types of sentences were produced; they consisted of noncase sentences as well as sentences containing secondary inferences, such as "The hot dogs were ready for the children." Their number was indirectly determined by the procedural constraint of writing exactly nine sentences. More importantly, because the mean number of such sentences across conditions was slightly greater than one, an analysis of possible differences was prevented by the probable floor effect.

To obtain an estimate of the reliability of the classification system, a second observer scored one protocol from each of four subjects in each condition. The correlation between the judgments of the two raters was .97. Therefore, the classifications made by the first observer were employed for all analyses.

Semantic accuracy. The mean number of sentences produced in the idea-set, inference, and elaboration categories were analyzed for 
Table 4

Mean Number of Sentences Produced in Each Condition, According to Sentence Category

\begin{tabular}{ccccc}
\hline & \multicolumn{4}{c}{ Condition } \\
\cline { 2 - 5 } Sentence category & $\begin{array}{c}\text { Bizarre } \\
\text { immediate }\end{array}$ & $\begin{array}{c}\text { Bizarre } \\
\text { delay }\end{array}$ & $\begin{array}{c}\text { Control } \\
\text { immediate }\end{array}$ & $\begin{array}{c}\text { Control } \\
\text { delay }\end{array}$ \\
\hline Idea set & 11.43 & 9.00 & 13.00 & 4.89 \\
Inference & 1.71 & 5.14 & 1.63 & 6.11 \\
Elaboration & .43 & 1.14 & .63 & 3.44 \\
Bizarre & 1.00 & .00 & .00 & .00 \\
\hline
\end{tabular}

differences among the four conditions, using the approach to nonorthogonal analysis of variance recommended by Appelbaum and Cramer (1974). Since none of the following analyses reliably interacted with the idea-set variable, the data were collapsed across idea sets or protocols. Table 4 contains the mean number of idea-set, inference, and elaboration sentences (as well as the mean number of bizarre sentences) that were produced in each condition.

The major findings were that subjects in both acquisition conditions wrote primarily idea-set sentences at immediate recall, whereas at delayed testing, the recall of control subjects not exposed to bizarre material was largely comprised of inference and elaboration sentences. The difference between acquisition conditions in immediate production of idea-set sentences was not reliable, $F(1,27)=2.22, M S_{\mathrm{e}}=9.28$, indicating that all subjects relied on information in the idea sets. Second, at delayed testing, the production of idea-set sentences declined for both acquisition conditions, but the decline was greater for the control condition, as indicated by a reliable interaction, $F(1,27)=6.67$, $M S_{\mathrm{e}}=9.28, p<.016$.

The mean number of inference sentences produced by both acquisition conditions clearly increased across testing conditions, $F(1,27)=11.69, M S_{\mathrm{e}}=10.63, p<.002$. To be certain that these inferences were based on memory for the presented material and were not artifactual of the frequency with which the cuing words might be combined arbitrarily into sentences with similar meaning, an additional group of 10 subjects was asked to construct 9 probable sentences for each set of cuing words. Out of the 180 sentences, only 7 inference-type sentences were produced. An informal comparison of the recall data to these production data indicates that inferences were reliably produced at delay.

Although the production of inference sentences did not reliably differ between acquisition conditions at delayed testing, controldelay subjects wrote significantly more elaboration sentences than did bizarre-delay subjects, $F(1,27)=6.01, \quad M S_{\mathrm{e}}=5.88$, $p<.021$. The tendency of bizarre-delay subjects to write more idea-set sentences and fewer elaboration sentences than controldelay subjects probably indicates better memory for the nature of the acquisition list and/ or a greater bias to write sentences that conform to it. In either case, hearing unusual sentences had a long-lasting effect on recall of sensible material.

Transformations of the bizarre. Four of the seven subjects in the bizarre-immediate condition wrote a total of seven sentences placed in the bizarre category. One subject attempted to preserve the bizarre quality of the acquisition sentence: "The bike was protective to the grandfather." The remaining six sentences were clear interpretations. Two of these contained the idea that the woman prepared hot dogs and occurred on protocols containing no other inferences. This type of inference was not written by subjects in other conditions. Therefore, these sentences may indicate transformational encoding. Furthermore, the other interpretations produced immediately (e.g., "The grandfather was happy that the bike was not hurt") were intuitively good examples of ef- 
fort after meaning. It is certainly possible that they were produced due to a bias to write sensible sentences, but since the design of the experiment logically reduced such a production bias, these sentences may at least partially indicate encoding efforts.

Virtually no evidence for transformations was produced at delayed testing, even though many inferences were written. By comparing these results to those of Experiment 1, it may be concluded that transformations were not sufficiently incorporated with information from the idea sets to be readily accessible at delayed testing. This line of reasoning also suggests that inference production occurred at testing; an inability to retrieve transformations of the bizarre implies a similar inability to retrieve previously stored inferences.

\section{General Discussion}

The more important results from Experiments 1 and 2 are relevant to Bartlett's (1932) assumption that unusual elements within a sensible context are omitted or transformed in an effort after meaning. These results demonstrate that the availability of bizarre information decreased dramatically over a 2-wk. period, and bizarre sentences were never recalled verbatim. Instead, they appear to have been transformed to fit the context of the sensible material. Transformations were produced in an immediate recall test, under conditions which should have minimized a sensible response bias. Furthermore, only the subjects who received bizzare input were reliably poorer at rejecting transformational distractors than general inference distractors at delayed recognition.

The last finding indicates some transformational representation of bizarre information, in spite of the findings that controldelay subjects tended to falsely recognize both types of inferential distractors and all subjects produced an increased number of inferences at delayed recall. Since bizarredelay subjects did not produce transformations at delayed recall, these inferences most likely resulted from processes operating during the memory test. At delayed testing, subjects presumably have less specific infor- mation available (Dooling et al., Note 1) and may, therefore, make inferences based on the general theme of the sensible material. The fact that bizarre-acquisition subjects did not use information contained in bizarre sentences to express these inferences at delayed recall certainly suggests that the transformational effect was relatively weak and that subjects did not incorporate transformations into their integrated representations for the sensible context. The nature of the acquisition task is possibly responsible for this weak effect. Bartlett (1932) found that unusual information was explained in a manner that facilitated comprehension of the entire passage, whereas the ideas employed in these experiments could be easily understood and inferences readily made. In short, materials of these experiments required fewer integrative efforts and less need to incorporate transformational attempts, compared to the processing of strange folktales.

Second, the results of both experiments suggest that the processing of syntactically strange information can improve memorial accuracy with regard to the sensible context. Although sensitivity to new distractors at immediate testing was not reliably superior for subjects who had heard bizarre sentences, these subjects made fewer positive responses to these distractors, compared to old sentences, than did control subjects. This pattern of results may very well indicate that bizarre information led to a more conservative response bias. Alternatively, the superior copying performance of bizarreacquisition subjects suggests that bizarre information affected encoding accuracy. The latter possibility, although unsupported by the $A^{\prime}$ data, is consistent with the view that the amount of cognitive effort employed to process at a particular level can account for differences in memory performance (Hertel, Tyler, McCallum, \& Ellis, Note 2). Either the raised response criterion explanation or the encoding effort explanation can be invoked to account for the superior recall accuracy of bizarre-delay subjects. After 2 wk., their performance demonstrated better memory for the nature of the acquisition list 
and fewer importations of nonimplicated details. It therefore appears that hearing syntactically unusual sentences leads to more cautious encoding and/or retrieval of the sensible context.

\section{Reference Notes}

1. Dooling, D. J., Christiaansen, R. E., \& Keenan, T. F. Levels of encoding and the forgetting of prose. Paper presented at the meeting of the Psychonomic Society, Denver, November 1975.

2. Hertel, P. T., Tyler, S. W., McCallum, M. C., \& Ellis, H. C. Cognitive effort in memory. Paper presented at the meeting of the Rocky Mountain Psychological Association, Denver, April 1978.

\section{References}

Appelbaum, M. I., \& Cramer, E. M. Some problems in the nonorthogonal analysis of variance. Psychological Bulletin, 1974, 81, 335-343.

Bartlett, F. C. Remembering: A study in experimental and social psychology. London, England: Cambridge University Press, 1932.

Bransford, J. D., Barclay, J. R., \& Franks, J. J. Sentence memory: A constructive versus interpretive approach. Cognitive Psychology, 1972, 3, 193-209.

Bransford, J. D., \& Franks, J. J. The abstraction of linguistic ideas: A review. Cognition, 1972, 1, 211-249.

Bransford, J. D., \& Johnson, M. K. Contextual prerequisites for understanding: Some investi- gations of comprehension and recall. Journal of Verbal Learning and Verbal Behavior, 1972, 11, 717-726.

Cofer, C. N. An historical perspective. In C. N. Cofer (Ed.), The structure of human memory. San Francisco: Freeman, 1976.

Graesser, A., \& Mandler, G. Recognition memory for the meaning and surface structure of sentences. Journal of Experimental Psychology: Human Learning and Memory, 1975, 104, 238248.

Green, D. M., \& Swets, J. A. Signal detection theory and psychophysics (2nd ed.). Huntington, N.Y.: Kreiger, 1974.

Jarvella, R. J. Syntactic processing of connected speech. Journal of Verbal Learning and Verbal Behavior, 1971, 10, 409-416.

Loftus, E. F. Leading questions and the eyewitness report. Cognitive Psychology, 1975, 7, 560-572.

Loftus, E. F., Miller, D. G., \& Burns, H. J. Semantic integration of verbal information into a visual memory. Journal of Experimental Psychology: Human Learning and Memory, 1978, 4, 19-31.

Pollack, I., \& Norman, D. A. A nonparametric analysis of recognition experiments. Psychonomic Science, 1964, 1, 125-126.

Sachs, J. S. Recognition memory for syntactic and semantic aspects of connected discourse. Perception \& Psychophysics, 1967, 9, 437-442.

Sulin, R. A., \& Dooling, D. J. Intrusion of a thematic idea in retention of prose. Journal of Experimental Psychology, 1974, 103, 255-262.

Received November 17, 1978 Revision received February 16, 1979 